\title{
Ranking risky alternatives: innovations in subjective utility analysis
}

\author{
J. W. Richardson \& J. L. Outlaw \\ Texas A\&M University, USA
}

\begin{abstract}
Over the past 50 years there have been innovations in the quantitative methods available to rank risky alternatives (mean-variance (MV), first degree stochastic dominance (FSD), and second degree stochastic dominance (SDS)). Two recent innovations, stochastic efficiency with respect to a function (SERF) and StopLight are compared to MV, FSD, and SDS to demonstrate the strengths and weaknesses of each method. The results indicate that SERF and Stoplight are powerful tools that do not suffer from some of the limitations as the other risk ranking methods.
\end{abstract}

Keywords: stochastic efficiency, stochastic dominance, mean variance, StopLight, certainty equivalent.

\section{Introduction}

The increased perception of risk in our world, coupled with the availability of microcomputers and simulation software, has made Monte Carlo simulation the most widely used methodology for analyzing risky systems and business situations. The popularity of Microsoft ${ }^{\circledR}$ Excel in business to answer simple "what if ..." questions and the availability of simulation add-ins, such as: Simetar,@Risk, and Crystal Ball, has increased the sophistication of analysis given to risky business decisions. Analysts with a strong foundation in Excel can easily learn how to turn their business spreadsheets into Monte Carlo simulation models with these risk analysis add-ins. In addition, short courses are available for those who want help getting started with incorporating Monte Carlo simulation into their Excel business spreadsheets.

After simulating alternative scenarios for a risky business decision the analyst is faced with the age old question of, "Which scenario is best?" In many 
instances, the analyst has hundreds or thousands of answers for each scenario, and mixed some place in the simulation results is the answer to the question. Some analysts give up and resort to simply ranking the risky scenarios based on their means, thus assuming the decision maker is risk neutral and negating the purpose of the Monte Carlo simulation.

A review of the literature on the topic of ranking risky alternatives offers risk analysts a number of tools that have been widely used and can be programmed into an Excel model. The more robust tools in the literature are mean-variance (MV) (Tobin [8]), first degree stochastic dominance (FSD) (Quirk and Saponik [11]), second degree stochastic dominance (SDS) (Hador and Russell [10]), and stochastic dominance with respect to a function (SDRF) (Meyers [3]). Each of these risk ranking tools has a following of risk analysts who extol their virtues; however, each has limitations that discourage its use in modern risk analysis.

Recently, Hardaker et al. [1] introduced a new method for ranking risky alternatives, stochastic efficiency with respect to a function (SERF). SERF overcomes the shortcomings of the risk ranking tools listed above. Another risk ranking tool that has proven useful when working with decision makers who lack statistical and economic training, but grasp the basics of probabilities, is a StopLight chart of target probabilities (Richardson et al. [7]).

\section{Objective}

The objective of this paper is to describe risk ranking methods: MV, FSD, SDS, and SDRF, SERF, and StopLight, and demonstrate how the six risk ranking tools can be applied to assist in decision making.

\section{Review of risk ranking tools}

Each of the six risk ranking tools introduced above is described and its attributes/ deficiencies are presented in this section.

\subsection{Mean variance (MV)}

The MV method compares the mean and variance for each risky alternative on the variance ( $\mathrm{Y}$ axis) and mean ( $\mathrm{X}$ axis) in Figure 1. The mean and variance for each risky alternative can be easily estimated from a Monte Carlo simulation model for the scenarios such as A, B, C, and D in Figure 1. The MV risk ranking tool assumes that the decision maker prefers the alternative with more income (mean) and lower risk (variance). In other words, the preferred scenario is in the south-east quadrant of a scenario with a lower income and higher variance alternative. For the risky alternatives in Figure $1, \mathrm{C}$ is preferred to $\mathrm{B}$. Alternative $\mathrm{C}$ is preferred to $\mathrm{D}$ because, for the same risk, $\mathrm{C}$ offers a greater mean. The deficiency of $\mathrm{MV}$ as a risk ranking tool is that both alternatives $\mathrm{A}$ and $\mathrm{C}$ are in the efficient (preferred) set because neither is in the south-east quadrant of another alternative. 


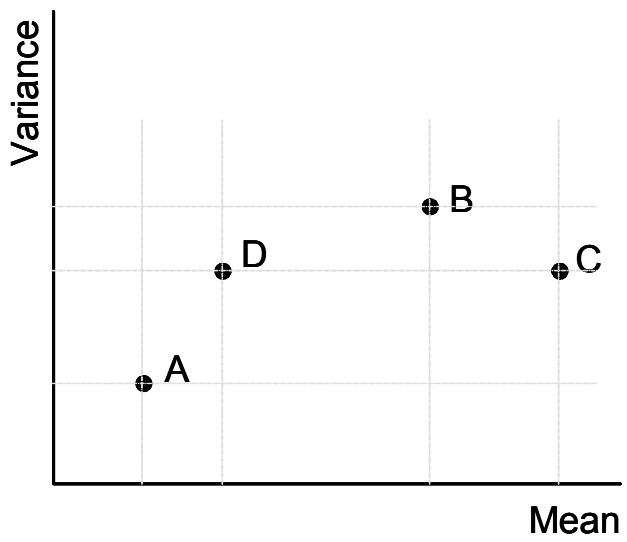

Figure 1: Mean variance ranking of risky alternatives.

\subsection{First degree stochastic dominance (FSD)}

The FSD risk ranking tool assumes that the decision maker prefers more (income or wealth) to less. The mathematical formula for FSD in the case of discrete observations generated by stochastic simulation models is:

$$
\sum \mathrm{F}(\mathrm{x}) \leq \sum \mathrm{G}(\mathrm{x}) \text { for all } \mathrm{x}
$$

where $\mathrm{F}(\mathrm{x})$ and $\mathrm{G}(\mathrm{x})$ are mutually exclusive cumulative distribution functions (CDF) representing risk alternatives $F$ and $G$. For $F(x)$ to dominate $G(x)$ at least one strong inequality must exist in (1). As indicated in Figure 2, F(x) lies below $\mathrm{G}(\mathrm{x})$ for each $\mathrm{x}_{\mathrm{i}}$, so $\mathrm{F}(\mathrm{x})$ is the preferred risky alternative, or we say $F(x)$ is FSD over $\mathrm{G}(\mathrm{x})$. The limitation of FSD is that, in real life, the CDFs for risky alternatives usually cross at least once. In that case FSD cannot be used for ranking risky alternatives. However, if the CDFs do not cross, FSD is the most robust risk ranking tool available.

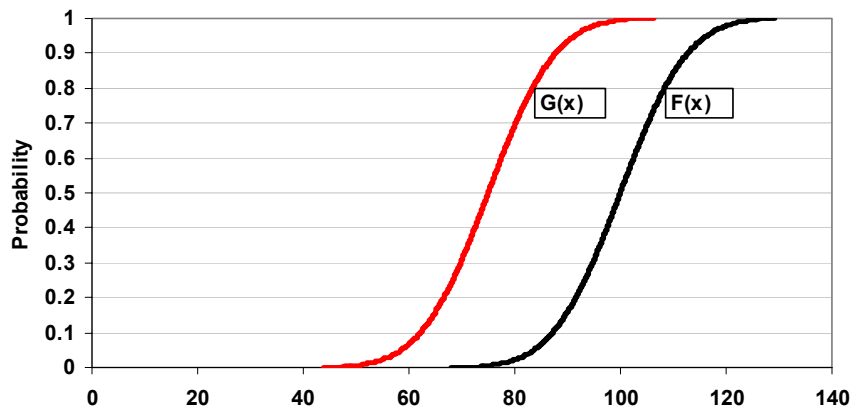

Figure 2: $\quad$ First degree stochastic dominance. 


\subsection{Second degree stochastic dominance (SDS)}

The SDS risk ranking tool assumes that the decision maker has constant absolute risk aversion and decreasing relative risk aversion (Hardaker et al. [2]). The mathematical formula for SDS holds that $F(x)$ is preferred to $G(x)$ if:

$$
\sum(F(x)-G(x)) \leq 0 \text { for all } x
$$

and the decision maker is indifferent between $F(x)$ and $G(x)$ if the sum is equal to zero. The advantage of SDS over FSD is that SDS can be used to rank pairwise risky alternatives whose CDFs cross one or more times. As indicated in Figure 3, SDS simply tabulates the sum of the differences between the CDFs or the sum of the vertical cross hatches minus the sum of the horizontal cross hatches. The deficiency for SDD is that it makes an assumption about the decision maker's risk preferences but does not take into consideration utility when ranking risky alternatives. Additionally, the methodology must be used to rank all possible pairs of risky alternatives, which can result in an efficient set with more than one scenario.

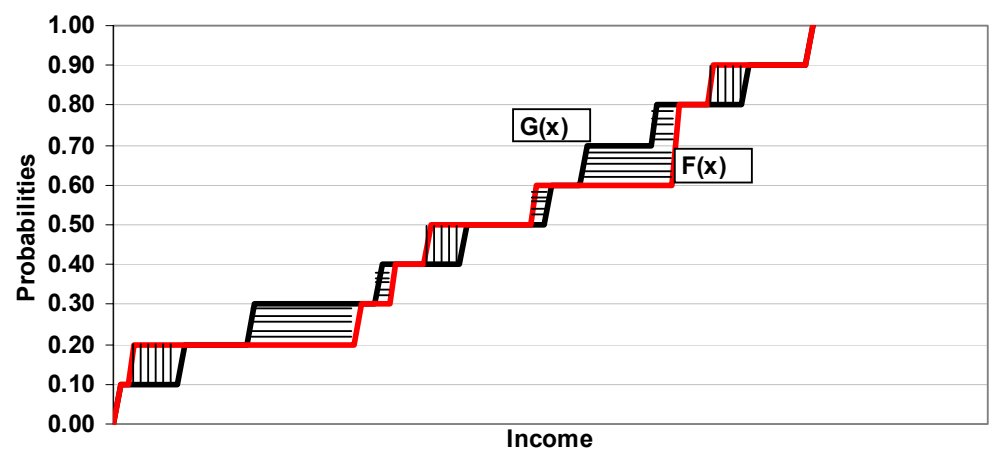

Figure 3: $\quad$ Second degree stochastic dominance.

\subsection{Stochastic dominance with respect to a function (SDRF)}

The SDRF tool for ranking risky alternatives extended the SDS method by incorporating utility. The mathematical formula for SDRF preferences of $\mathrm{F}(\mathrm{x})$ over $\mathrm{G}(\mathrm{x})$ in the discrete case is:

$$
\sum U\left(x, r_{i}(x)\right) F(x) \leq \sum U\left(x, r_{i}(x)\right) G(x)
$$

Equation (3) is evaluated for each $\mathrm{x}_{\mathrm{i}}$ using a lower risk aversion coefficient (RAC) $\left(\mathrm{r}_{\mathrm{L}}(\mathrm{x})\right)$ and using an upper RAC $\left(\mathrm{r}_{\mathrm{U}}(\mathrm{x})\right)$. The lower and upper RACs are chosen based on estimates of the decision maker's range of risk aversion. The SDRF can result in an efficient set with more than one risky alternative if the decision maker's preferences are different for the lower and upper RAC (McCarl [5]). This deficiency and the fact that SDRF has to be run for all possible pairwise combinations of the risky alternatives makes SDRF computationally 
difficult and may not result in a small efficient set. The computer code for SDRF is quite complicated and is not easily programmed; however, it is available in the Simetar add-in to Excel. (Simetar(C) is an Excel add-in for simulation, econometrics, and ranking risky alternatives developed by Richardson et al. [4]. Additional information about Simetar is available at www.simetar.com.)

\subsection{Stochastic efficiency with respect to a function (SERF)}

The SERF tool introduced by Hardaker et al. [1] is a more transparent method to rank risky alternatives than SDRF. SERF calls for calculating the certainty equivalent (CE) for a number of $\mathrm{r}_{\mathrm{i}}(\mathrm{w}) \mathrm{s}$ between a lower and upper range of RACs. The CE for a risky alternative is the certain sum of $w$ that has the same utility as the expected utility of the risky alternative from (4). The inverse utility function can be evaluated at each $r_{i}(w)$ for each risky alternative, $j$, to calculate the $\mathrm{CE}_{\mathrm{ij}}$ used in the ranking by evaluating the following formula:

$$
\mathrm{CE}\left(\mathrm{w}_{\mathrm{ij}}, \mathrm{r}_{\mathrm{i}}(\mathrm{w})\right)_{\mathrm{ij}}=\mathrm{U}^{-1}\left(\mathrm{w}_{\mathrm{ij}}, \mathrm{r}_{\mathrm{i}}(\mathrm{w})\right)
$$

where $\mathrm{U}^{-1}(\cdot)$ is the inverse utility function.

The result is an IxJ table of CEs (with I rows for the $r_{i}(w) s$ evaluated between $r_{L}(w)$ and $r_{U}(w)$ and $J$ columns for the $J$ risky alternatives). Assuming the decision maker prefers more utility to less, the risky alternative with the greatest $\mathrm{CE}$ in each row is preferred by decision makers who possess that level of risk aversion. To facilitate decision making the SERF table is converted into a chart, as depicted in Figure 4. In this figure, we see that Alternative 1 is preferred for all decision makers with $\mathrm{r}(\mathrm{w})$ less than $\mathrm{r}_{2}(\mathrm{w})$, and for decision makers more risk averse than $r_{2}(w)$, the preferred risky alternative would be Alternative 2 .

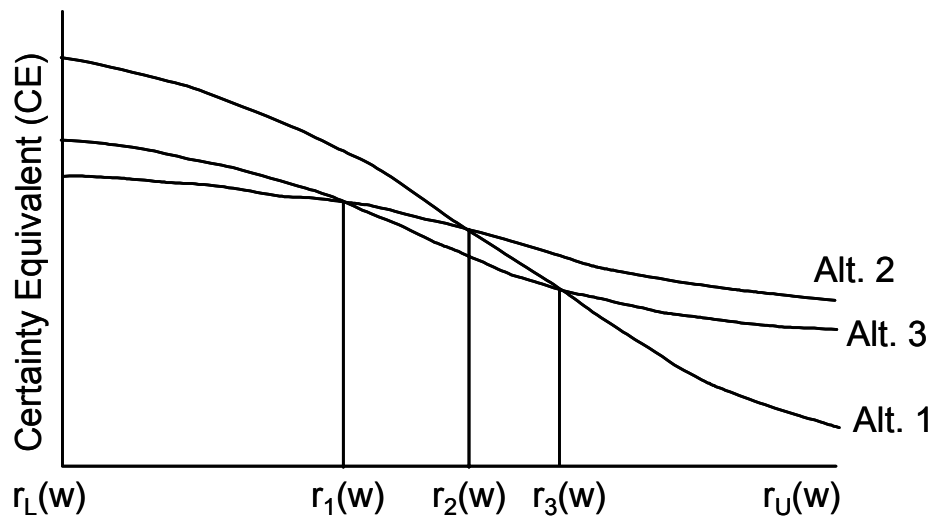

Risk Aversion

Figure 4: SERF ranking of risky alternatives. Source: Hardaker et al. [1]. 
One advantage of SERF over SDRF is that the analyst does not have to know the decision maker's $r(w)$. By setting $r_{L}(w)$ equal to zero (risk neutral) and $r_{U}$ (w) at a value consistent with an extremely risk averse decision maker, the SERF analysis ranks risky alternatives for all types of decision makers. The risk rankings can then be identified by classes of decision makers. Anderson and Dillon [6] proposed the following schedule for $\mathrm{r}(\mathrm{w})$, thus classifying risk averse decision makers:

$\begin{array}{ll}\text { Risk neutral } & 0 \\ \text { Slightly risk averse } & 0.5 \\ \text { Normally risk averse } & 1 \\ \text { Moderately risk averse } & 2 \\ \text { Extremely risk averse } & 4\end{array}$

\begin{tabular}{l} 
Absolute a(w) \\
\hline 0 \\
$0.5 / \mathrm{w}$ \\
$1 / \mathrm{w}$ \\
$2 / \mathrm{w}$ \\
$4 / \mathrm{w}$
\end{tabular}

(Note: The second column for absolute $\mathrm{r}(\mathrm{w})$ was added by the authors using the formula that absolute risk aversion $\mathrm{a}(\mathrm{w})$ equals relative risk aversion $(\mathrm{r}(\mathrm{w})$ ) divided by wealth (w).)

The second advantage of SERF over SDRF is that it ranks all risky alternatives simultaneously. The most obvious advantage is that the SERF rankings can be presented graphically so decision makers not familiar with expected utility can easily see which alternative is preferred for their range of risk aversion. The SERF risk ranking tool can be used with any inverse utility function and can be used by risk preferring and risk averse decision makers. SERF is included in Simetar, so its implementation is easy. Regarding the utility function selection, annual decisions based on income rather than wealth generally use the negative exponential utility function with absolute risk aversion coefficients. While multiple year analyses that rely on an ending wealth use the power utility function with relative risk aversion coefficients.

\subsection{StopLight charts}

The authors' research on farm policy for the U.S. Congress often requires ranking alternative policy scenarios based on their impacts on farmers' economic viability. The risk ranking tools described in sections 3.1-3.5 are not easily understood by the majority of the policy makers. (The few economists who advise policy makers have used SERF rankings but do not rely on MV, FSD, SDS or SDRF rankings. The majority of staffers who advise policy makers are not versed in the risk ranking tools in sections 3.1-3.5 but they have a basic understanding of probabilities.) To communicate the rankings of alternative policies to policy makers the authors developed StopLight Charts (Figure 5). The charts display the probabilities for a favorable outcome and an unfavorable outcome for each policy as different colors in a stacked bar chart. The probability of achieving a favorable outcome is denoted as green. The probability of an unfavorable outcome is shown as red in each bar. The probability of values between the favorable and unfavorable outcomes are depicted in yellow. 


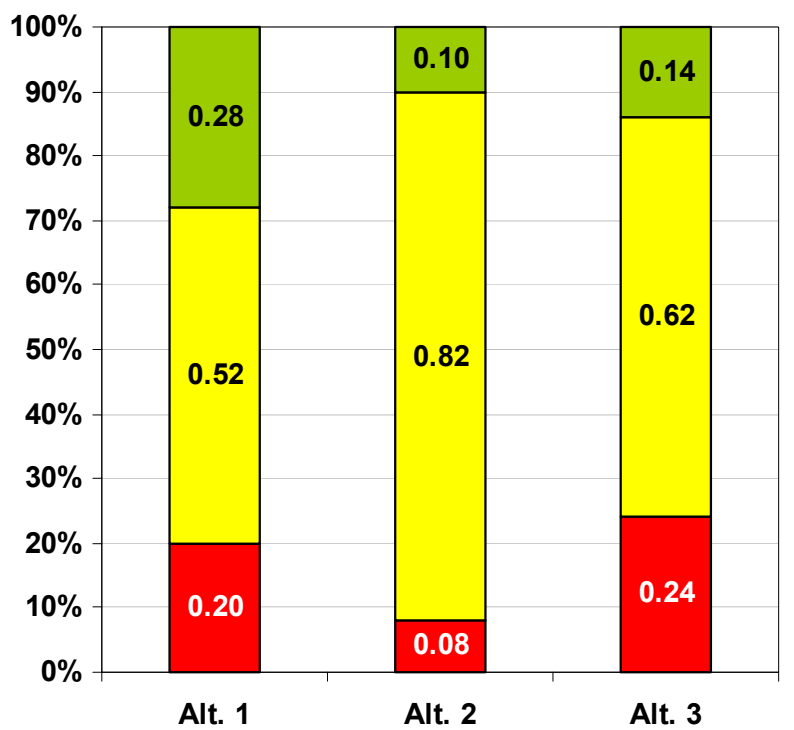

Figure 5: StopLight to rank three risky alternatives.

Interpretation of the StopLight Chart is quite simple. The preferred risky alternative is the one with the least red and the most green for normal risk averse decision makers. The ranking rule is consistent with SERF, SDRF, and SDS in that the decision maker prefers the scenario with the smallest utility weighted mass of unfavorable outcomes and the greatest utility weighted mass of good outcomes. The advantages of StopLight Charts over other risk ranking tools are that it is easy to calculate, no assumption about the decision maker's degree of risk aversion or utility function is required, and the analyst does not make the ranking. The decision maker ranks the risky alternatives based on his/her own utility function for income and risk after setting minimum and maximum target outcome levels and observing the green and red probabilities in the alternative bars.

\section{Stochastic model to demonstrate risk ranking tools}

The stochastic simulation model used to demonstrate the risk ranking tools is depicted in Figure 6 . The model consists of equations to simulate the endogenous variables $\mathrm{Y}_{\mathrm{it}}$ that are a function of stochastic variables $\left(\mathrm{X}_{\mathrm{it}}\right)$, exogenous variables $\left(\mathrm{E}_{\mathrm{it}}\right)$, assumed input values $\left(\mathrm{Z}_{\mathrm{it}}\right)$, and the manager's controls $\left(\mathrm{C}_{\mathrm{ij}}\right)$ that define $\mathrm{J}$ alternative scenarios. The $\mathrm{t}$ subscript indicates that the model can be a recursive intertemporal model of a stochastic process. The form of the model used for this article is a multiple-year financial model with its equations defined by the pro forma financial statements for income, cash flows, and balance sheet. 


\section{Stochastic Variables}

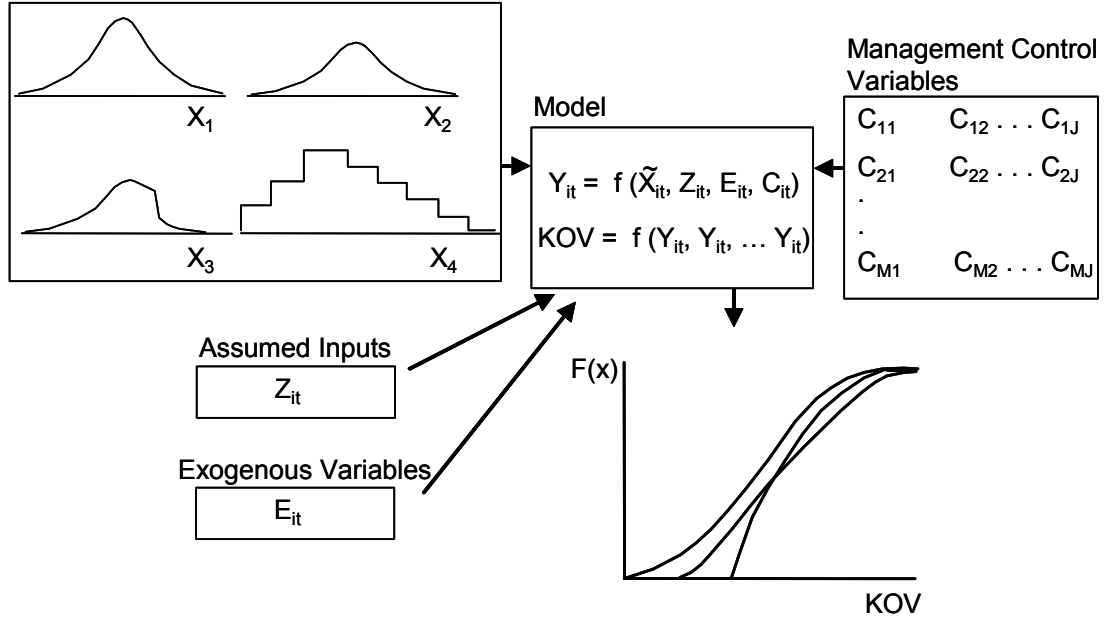

Figure 6: Schematic of a stochastic simulation model for analyzing $\mathrm{J}$ scenarios over $\mathrm{M}$ control variables.

The outputs for the model are the CDFs of the key output variable (KOV) for the model. For the multiple-year financial model used here, the KOV is the firm's net present value (NPV) as this variable incorporates the present value of changes in wealth over a $\mathrm{T}$ period planning horizon and the present value of annual net income.

Stochastic variables for the model are the annual prices for inputs, the annual level of production, and the prices for the products produced. The control variables are different combinations of the firm's product mix, marketing strategies, and production insurance.

Table 1: $\quad$ Summary statistics for four risky alternatives.

\begin{tabular}{lccrc}
\hline & NPV 1 & NPV 2 & NPV 3 & NPV 4 \\
\hline Mean & 86,546 & 91,338 & 72,058 & 93,320 \\
Std Dev & 139,402 & 103,109 & 131,642 & 124,935 \\
Coef Variat & 161 & 113 & 183 & 134 \\
Minimum & $(339,045)$ & $(273,801)$ & $(363,982)$ & $(335,810)$ \\
Maximum & 490,458 & 382,024 & 439,190 & 454,823 \\
\hline
\end{tabular}

\section{Results for alternative risk ranking tools}

Summary statistics for the simulated NPV empirical probability distributions are summarized in Table 1. Scenario 4 has the largest mean; however, Scenario 2 has the lowest standard deviation and Scenario 3 has the largest maximum, so it is not feasible to determine the ranking from the summary statistics. The MV 
ranking is presented in Figure 7. Based on the southeast quadrant rule, Scenario 4 is preferred to Scenarios 1 and 3. The efficient contains both Scenarios 2 and 4 based on the MV ranking tool.

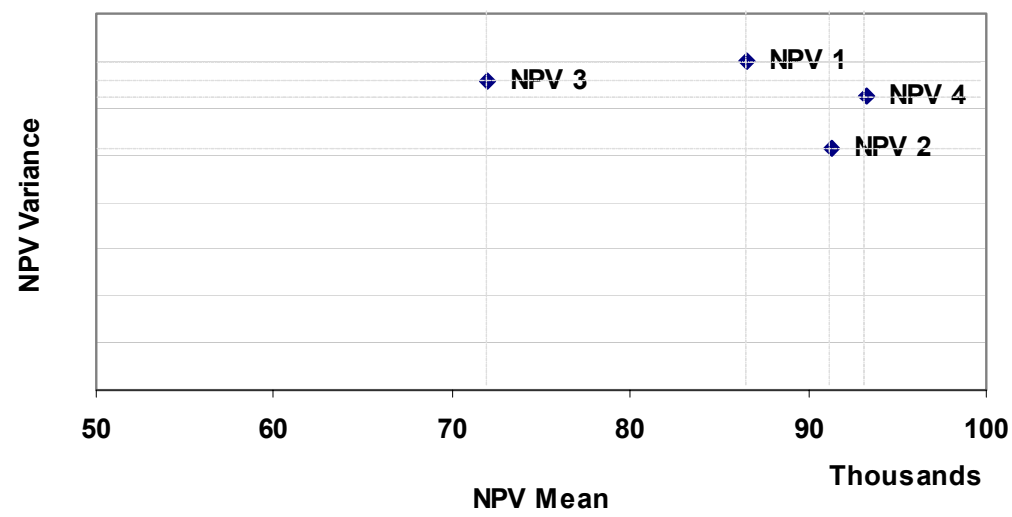

Figure 7: $\quad$ MV ranking of four risky alternatives.

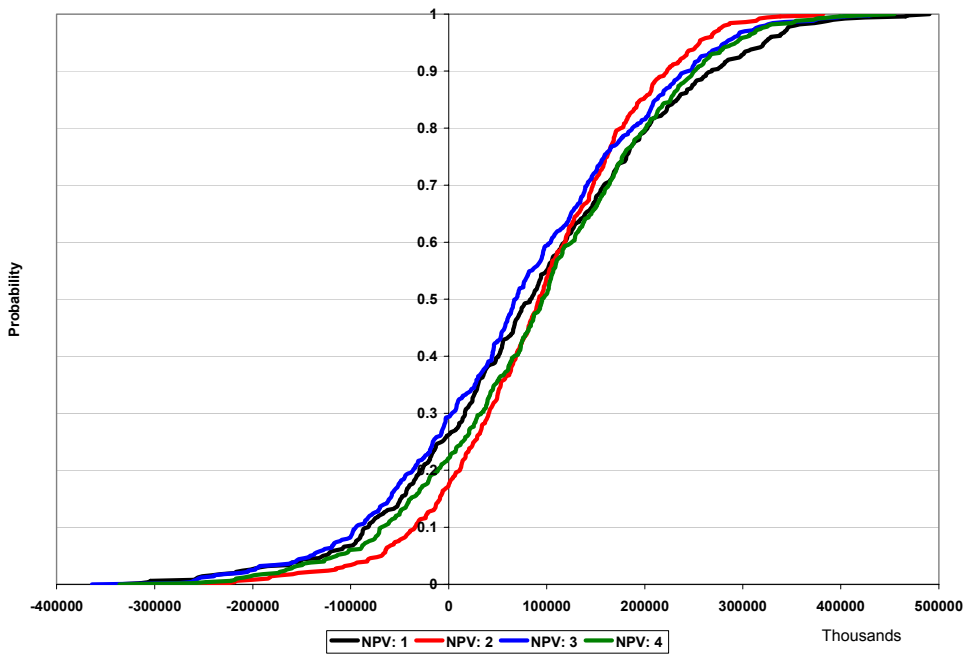

Figure 8: CDFs of four risky alternatives.

Figure 8 displays the CDFs for the four risky alternatives. Each of the CDFs cross at least once, so it is not feasible to rank the scenarios by FSD.

Table 2 summarizes the FSD, SDS, and SDRF rankings provided by Simetar. As expected from the CDF chart, none of the scenarios are FSD preferred. The SDS ranking suggests that Scenario 4 dominates Scenarios 1, 2, and 3, resulting in only one scenario in the efficient set. The SDS results also indicate that if 
Scenario 4 is not feasible, the decision maker would prefer Scenario 2. The results for SDRF, also in Table 2, indicate that a risk averse decision maker with lower and upper absolute risk aversion coefficients of 0 to 0.00000667 (the upper absolute risk aversion coefficient represents a person who is extremely risk averse and is calculated as $\mathrm{a}(\mathrm{w})=4.0 / 600,000$ where 600,000 is net worth for the decision maker) would be indifferent between Scenarios 4 and 2. The SDRF risk ranking tool used the negative exponential utility function. Decision makers who are risk neutral prefer Scenario 4 while those who are extremely risk averse prefer Scenario 2. Unfortunately, SDRF does not provide any further specification in the ranking.

Table 2: $\quad$ Ranking risky alternatives based on FSD, SDS, and SDRF.

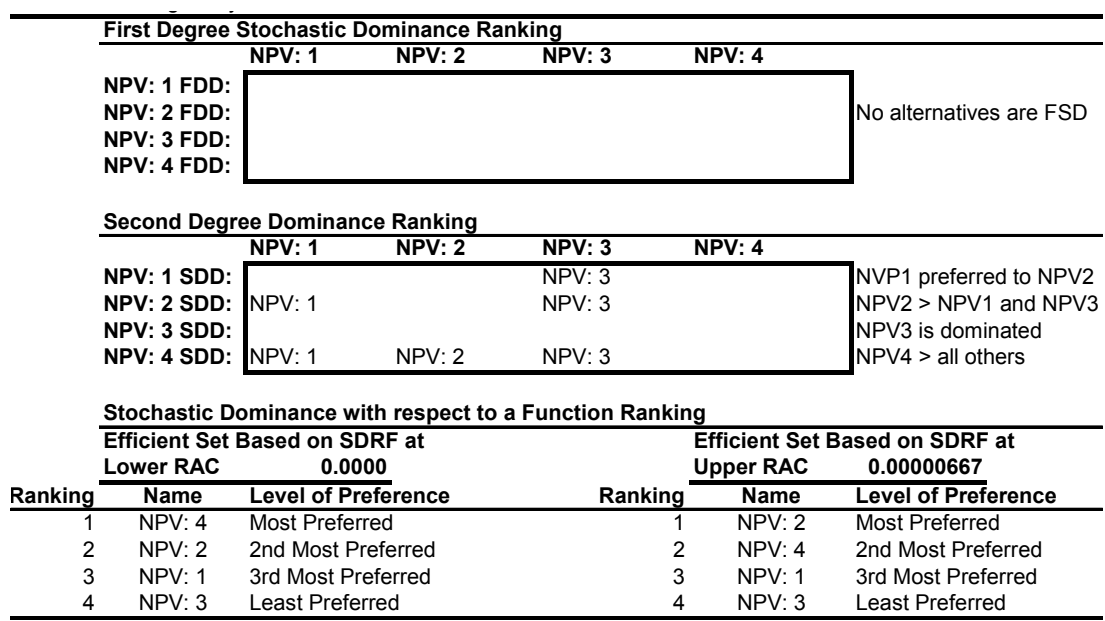

The SERF ranking is presented in Figure 9. The SERF chart shows that all decision makers with a $\mathrm{r}(\mathrm{w})$ less than 0.5 prefer Scenario 4. For all decision makers who exhibit normal, moderate, or extreme risk aversion, the preferred scenario is number 2 . The SERF rankings used the power utility function with $\mathrm{r}(\mathrm{w}) \mathrm{s}$ because the distributions represent income and wealth changes over a multiple-year time frame. Hardaker et al. [1] indicated that the difference between CEs at a given $\mathrm{r}(\mathrm{w})$ represent the decision maker's risk premium of the preferred scenario over a less preferred risky alternative. As one would expect, the risk premium between Scenario 2 and the less preferred scenarios increases as the decision maker becomes more risk averse.

Figure 10 presents a StopLight chart for lower and upper target values of NPV less than zero or greater than $\$ 150,000$. The lower target value is consistent with the decision maker wanting to earn a return greater than the discount rate, i.e., a positive NPV. The upper target can be set by the decision maker as Simetar dynamically updates the chart for alternative targets. The StopLight chart ranking agrees with the SERF analysis. Scenario 2 is preferred 


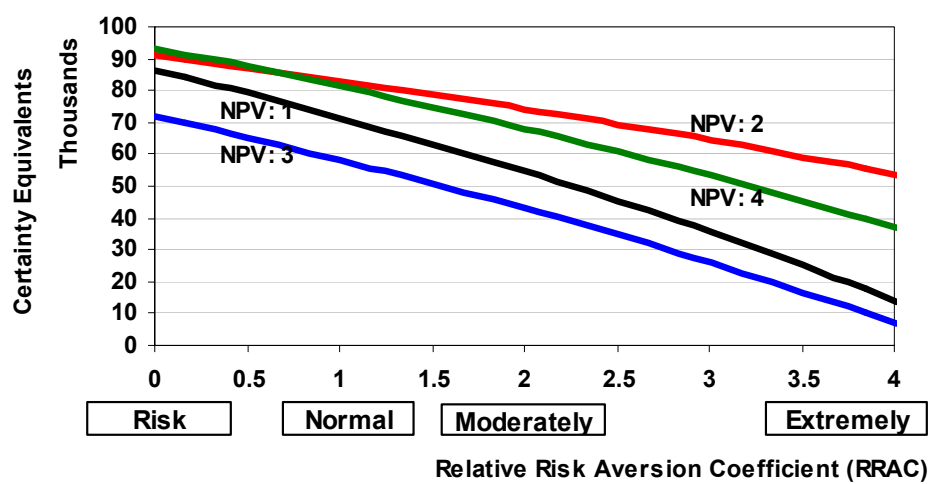

Figure 9: $\quad$ SERF ranking of four risky alternatives.

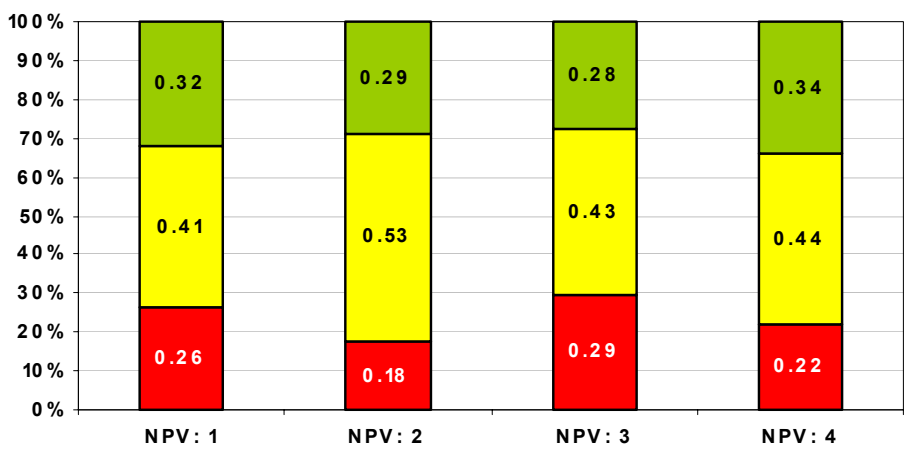

Figure 10: StopLight chart to rank four risky alternatives.

by risk averse decision makers because it has the lowest probability of a negative NPV. Decision makers who are risk neutral to only slightly risk averse would prefer Scenario 4 because it has the highest probability of exceeding the upper target. The advantage of the StopLight chart for ranking risky alternatives is that the analyst can ask the decision maker for his/her lower and upper targets and then let them decide which scenario is best. No utility function or risk aversion coefficient has to be elicited and the decision maker makes the decision based on values he/she is familiar with in the context of the problem at hand.

\section{Summary and conclusions}

The art and science of quantitatively ranking risky alternatives has progressed in discrete jumps over the past fifty years. The latest advances offered by SERF and StopLight charts are transparent and provide the analyst with more concrete evidence to explain rankings than previous analytical tools. 


\section{References}

[1] Hardaker, J.B., J.W. Richardson, G. Lien, K.D. Schumann. "Stochastic Efficiency Analysis with Risk Aversion Bounds: A Simplified Approach." The Aust. J. Agric and Res. Econ, 48(2004b): 253-270.

[2] Hardaker, J.B., R.B.M. Huirne, J.R. Anderson, and G. Lien. Coping With Risk in Agriculture. $2^{\text {nd }}$ ed. CAB International, Wallingford (2004a).

[3] Meyers, J. "Choice Among Distributions." J. Econ Theory. 14(1977): 326-336.

[4] Richardson, J.W., K.D. Schumann, and P. Feldman. "Simetar: Simulation and Econometrics to Analyze Risk." College Station, Texas, 2005.

[5] McCarl, B.A. "Preferences Among Risky Prospects Under Constant Risk Aversion." S. J. of Agric. Econ. 20(1988): 25-33.

[6] Anderson, J.R. and J.L. Dillon. Risk Analysis in Dryland Farming Systems. Farming Systems Management Series No. 2. Rome: FAO, 1992.

[7] Richardson, J.W., J.L. Outlaw, G.M. Knapek, J.M. Raulston, B.K. Herbst, R.J. Fumasi, D.P. Anderson, and S.L. Klose. "Representative Farms Economic Outlook for the January 2007 FAPRI/AFPC Baseline." Texas Agricultural Experiment Station, Texas Agricultural Extension Service, Texas A\&M University, Department of Agricultural Economics, Agricultural and Food Policy Center Briefing Paper 07-2, February 2007. http:/www.afpc.tamu.edu/pubs/3/463/BP\%2007-2\%20-20for\%20web.pdf

[8] Tobin, J. "Liquidity Preference as Behavior Towards Risk." Rev of Econ Studies. 25(1958): 65-86.

[9] Hansch, G. and H. Leuy. "The Efficiency of Choices Involving Risk." Rev of Econ Studies. 36(1969): 335-346.

[10] Hador, J. and W.R. Russell. "Rules for Ordering Uncertain Prospects." Amer. Econ. Rev. 59(1969): 25-34.

[11] Quirk, J. and R. Saposnik. "Admissibility and Measurable Utility Functions." Rev of Econ Studies. 29(1962): 140-146. 